\title{
Exome sequencing in congenital ataxia identifies two new candidate genes and highlights a pathophysiological link between some congenital ataxias and early infantile epileptic encephalopathies
}

\author{
Stéphanie Valence, MD, Lydie Burglen, MD, PhD et al."
}

Purpose: To investigate the genetic basis of congenital ataxias (CAs), a unique group of cerebellar ataxias with a nonprogressive course, in 20 patients from consanguineous families, and to identify new CA genes.

Methods: Singleton -exome sequencing on these 20 well-clinically characterized CA patients. We first checked for rare homozygous pathogenic variants, then, for variants from a list of genes known to be associated with CA or very early-onset ataxia, regardless of their mode of inheritance. Our replication cohort of 180 CA patients was used to validate the new CA genes.

Results: We identified a causal gene in 16/20 families: six known CA genes (7 patients); four genes previously implicated in another neurological phenotype (7 patients); two new candidate genes (2 patients). Despite the consanguinity, $4 / 20$ patients harbored a heterozygous de novo pathogenic variant.
Conclusion: Singleton exome sequencing in 20 consanguineous CA families led to molecular diagnosis in $80 \%$ of cases. This study confirms the genetic heterogeneity of CA and identifies two new candidate genes (PIGS and SKOR2). Our work illustrates the diversity of the pathophysiological pathways in CA, and highlights the pathogenic link between some CA and early infantile epileptic encephalopathies related to the same genes (STXBP1, BRAT1, CACNA1A and CACNA2D2).

Genetics in Medicine (2019) 21:553-563; https://doi.org/10.1038/s41436018-0089-2

Keywords: Congenital ataxia; exome sequencing,; Cerebellar atrophy; Early infantile epileptic encephalopathies; Pathophysiology

\section{INTRODUCTION}

Congenital ataxias (CAs) are a unique group of cerebellar ataxias that were first described in 1893 by Freud and in 1903 by Batten. They are characterized by the occurrence of very early cerebellar symptoms in children whose psychomotor skills improve thereafter, and are supposed to be more developmental than degenerative diseases. ${ }^{1-3}$ CAs are characterized by an absence of degeneration during the course of the disease and are supposed to be nonprogressive. They are different from early-onset cerebellar ataxia as defined by Harding in 1981 as an unremitting, progressive ataxia with an onset in the two first decades. ${ }^{4}$ However, in some cases, it may be difficult to determine whether the course of the disease is progressive or nonprogressive, due to the advances made by the child during this period of active development, particularly if degeneration is slow and the onset very early. ${ }^{5}$

Hypotonia and delayed motor development, becoming apparent during the first or second year of life, are the first nonspecific signs of $\mathrm{CA}$, preceding the appearance of ataxia, which may be associated with intellectual deficiency (ID), epilepsy. and other neurological or ophthalmological symptoms. ${ }^{5}$ Brain magnetic resonance image (MRI) is an essential tool for the diagnostic evaluation of $\mathrm{CA}^{6}{ }^{6}$ MRI may be normal, but, in most cases, it reveals cerebellar abnormalities (dysplasia, hypoplasia, or atrophy), nonspecific or pathognomonic features, as in the Joubert and Dandy-Walker syndromes. Most etiologies of CA seem to be genetic, but genetic investigations should be preceded by the exclusion, by clinical evaluation, MRI and standard metabolic screening, of nongenetic early-onset ataxias (e.g., ischemic, hemorrhagic, toxic, infectious, and tumoral ataxias) and inborn errors of metabolism leading to ataxia, such as mitochondrial, glycosylation, or lysosomal disorders. ${ }^{5,7}$

To date, about 30 genes have been involved in "congenital ataxia," defined as this at least apparently nonprogressive ataxia, symptomatic before two years of life. Among them, a few genes are in fact responsible for a progressive ataxia, but with a very slow course and a very early onset during the first

Correspondence: Lydie Burglen lydie.burglen@aphp.fr). " A full list of authors and their affliations appears at the end of the paper. 
2 years, compatible with progress in the child, and are clinically indistinguishable from CA at least in the first years (for example, SNX14, SACS). The CA genes encode proteins involved in several pathophysiological pathways or mechanisms, including glutamate metabolism (GRM1, SPTBN2, and GRID2), channels (CACNA1A, ITPR1, CA8, KCNJ10, and KCNC3), mitochondria (SACS, PMPCA, and COQ8A/ $A D C K 3)$, neuronal migration $(V L D L R)$, chaperone proteins (SIL1), regulators of autophagy and endocytic trafficking (RUBICON), and autophagosome lysosome dysfunction (SNX14); or that have no clearly understood pathological mechanism (WDR81, ATP8A2, ABCB7, ATCAY and $A T P 2 B 3)$. Nevertheless, many patients with CA still have no molecular diagnosis; most of the published series have not been focused on this specific group, and have addressed "ataxia" or "cerebellar atrophy" without taking into account both the age of onset and the clinical phenotype (progressive or not). ${ }^{6,8-10}$

To identify new genetic causes of CA, we performed singleton exome sequencing (ES) on 20 unrelated CA patients (with no evidence of progression at the time of inclusion) from consanguineous families. We present the pathogenic or likely pathogenic variants that we believe can account for the CA phenotype in $16(80 \%)$ of the patients, together with clinical and radiological data for these patients.

\section{MATERIALS AND METHODS}

\section{Patients}

We recruited 200 unrelated CA patients from the French Reference Centre for Cerebellar Malformations and Congenital Diseases. All patients presented clinical cerebellar symptoms before the age of 2 years. We excluded patients whose ataxia was clearly progressive at the time of inclusion, patients with chromosomal anomalies explaining the disorder (karyotype and/or single-nucleotide polymorphism [SNP] array), classical metabolic disorders, and patients whose brain MRI results were suggestive of a specific diagnosis, such as Joubert and Dandy-Walker syndromes, VLDLR pathogenic variants $(\mathrm{PV})$, acquired cerebellar damage, or leukoencephalopathies.

From these 200 patients, we selected a subgroup of 20 probands (aged from 4 to 20 years) from 20 unrelated consanguineous families (19 simplex and 1 multiplex families). We carried out a detailed analysis of clinical features and brain MRI. Blood samples were obtained from the index cases, their parents, and, when available, healthy siblings, after written informed consent had been obtained, in accordance with our institution's ethics policy for human studies (Ile de France IX Ethics Committee approval granted to L. Burglen, IDRCB 2010-A00715-34).

\section{ES and filtering data}

Given the consanguinity of these families, we used a strategy based on a combination of homozygosity mapping and ES for the proband only (Supplementary Materials and Methods). Indeed, we first considered homozygous variants because of the presumed autosomal recessive mode of inheritance, and DNA variants were prioritized according to the following criteria: high quality score (Q Phred score $\geq 30$ ) and coverage $\geq 20 \times$ in probands, minor allelic frequency (MAF) $<0.01$, conservation and predicted impact on coding and noncoding sequence using the Alamut biosoftware, presence in ClinVar. ${ }^{11}$ Among the genes harboring the filtered variants, we then selected the strongest candidate genes, as follows: (1) genes known to be associated with CA or ataxia; (2) genes known to be associated with other neurological phenotypes in humans, suggesting a phenotypic expansion; (3) genes not known to be associated with human disease but considered to be strong candidates for involvement in CA on the basis of comprehensive literature reviews and database analyses; (4) noncandidate or unknown genes considered to require further validation. If no clear candidate gene was identified after this initial filtering step, we considered, despite consanguinity, the possibility of autosomal recessive inheritance with compound heterozygous variants, as well as autosomal dominant or Xlinked mode of inheritance, and enlarged our strategy to encompass heterozygous and X-linked hemizygous variants, beginning with the list of all genes known to be involved in $\mathrm{CA}$, as well as some genes responsible for very early-onset slowly progressive ataxia (Table S1).

\section{Variant confirmation, segregation analysis, replication cohort}

All putative pathogenic variants were confirmed by Sanger sequencing of DNA from the affected children, their parents, and family members, when available, by standard protocols. If a de novo variant was found, the parentage of the child was checked with 16 highly polymorphic markers (PowerPlex ${ }^{\circledR}$ 16 HS System, Promega). For the five candidate genes whose validation required further arguments (BRAT1, CACNA2D2, STXBP1, SKOR2, and PIGS), we performed targeted nextgeneration sequencing (NGS) on the other 180 patients of our CA cohort (Supplementary Materials and Methods). Pathogenicity of the variants was ascertained according to the American College of Medical Genetics and Genomics-Association for Molecular Pathology (ACMG/ AMP) recommendation. ${ }^{12}$

\section{RESULTS}

Identification of novel variants in six genes known to be associated with CA (WDR81, SNX14, SACS, ITPR1, STXBP1, and CACNA1A)

In 7 of the 20 probands (35\%), we identified causal variants of six genes known to be involved in CA or very early-onset slowly progressive ataxia. In three patients, the pathogenic variant was homozygous, consistent with the suspected autosomal recessive mode of inheritance (genes WDR81, SACS, and SNX14) 13-15. However, despite the consanguinity, we found no convincing homozygous variants in patients P4, P5, P6, and P16. Filtering for heterozygous variants in genes from our candidate genes list and highly pathogenic variants allowed the identification of heterozygous pathogenic 

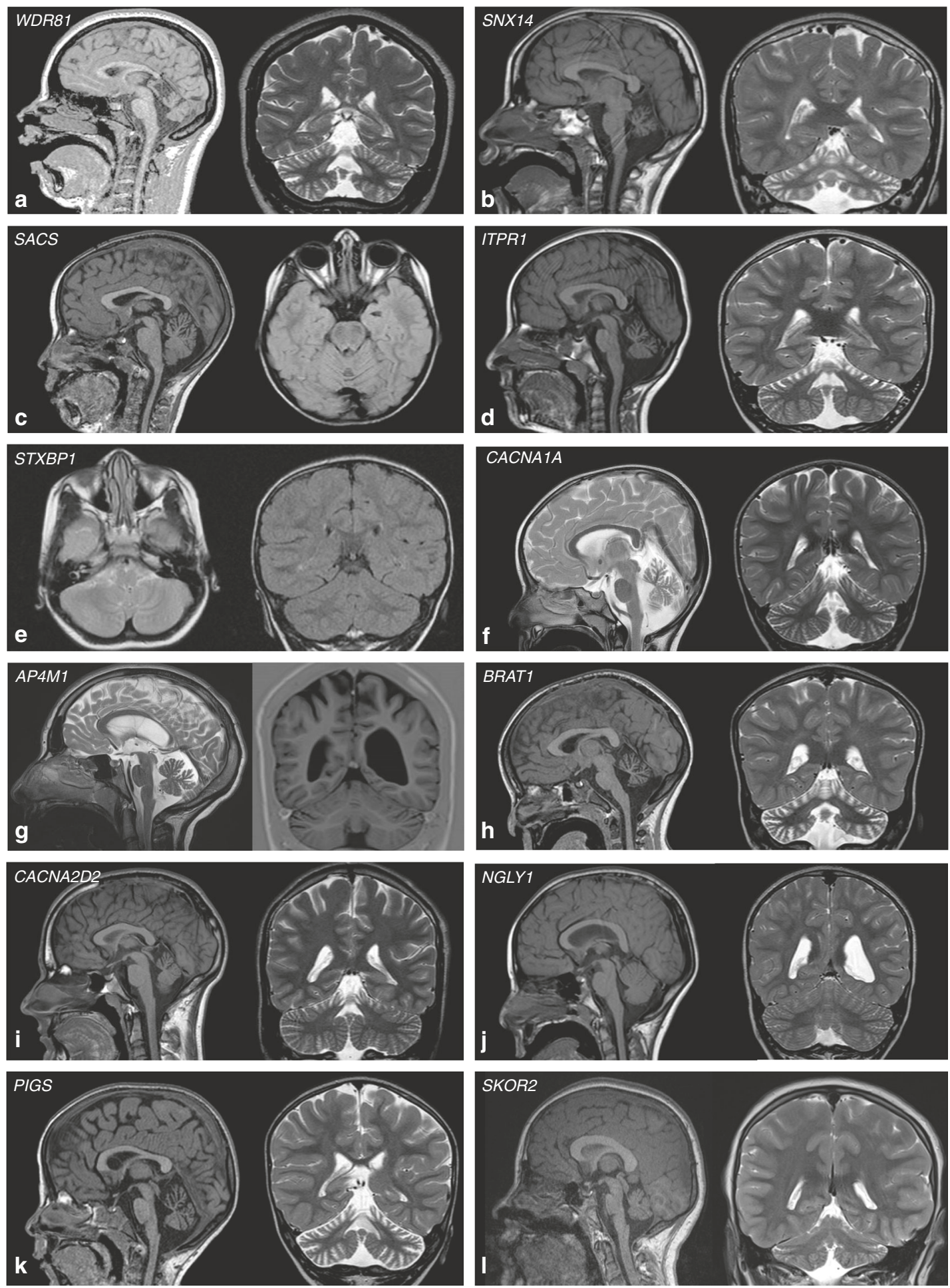

variants of ITPR1 (2 patients), CACNA1A (1 patient), and STXBP1 (1 patient). ${ }^{16-18}$ The analysis of parental DNA showed that these four variants have occurred de novo. The clinical data, genotypes, and pedigrees of the seven patients are described in the supplemental data (Table S2, Table S3 for ACMG/AMP classification, Fig. S1). MRI showed variable nonspecific atrophy of the vermis and cerebellar hemispheres, with the exception of patient P3 in whom axial fluid attenuation inversion recovery (FLAIR) images revealed linear hypointensities of the pons suggestive of Charlevoix-Saguenay ataxia, and STXBP1-mutated patient P6 who had a normal MRI (Fig. 1). Heterozygous variants of STXBP1 cause early infantile epileptic encephalopathy-4 (EIEE4, MIM 612164), characterized by severe epilepsy, 
Fig. 1 Magnetic resonance image (MRI) features of the patients with identified causal or strongly candidate pathogenic variants. a WDR81: P1 (17 years): midsagittal T1-weighted sequence showing a small vermis with enlargement of interfolium spaces (anterior lobe) and an enlarged primary fissure. Note the thin splenium of the corpus callosum. Coronal T2-weighted image showing a slight diffuse enlargement of the interfolium spaces of the cerebellar hemispheres. b SNX14: P2 (10 years): midsagittal T1-weighted sequence and coronal T2-weighted image showing a small vermis and diffuse enlargement of the interfolium spaces in both the cerebellar vermis and hemispheres. c SACS: P3 (6 years): midsagittal T1-weighted sequences showing enlargement of interfolium spaces (anterior lobe and rostral part of the posterior lobe). Axial FLAIR images showing linear hypointensities of the pons. $\mathbf{d}$ /TPR1: P5 (5 years): midsagittal T1-weighted sequences showing a slightly undersized vermis with enlargement of the interfolium spaces (anterior lobe and rostral part of the posterior lobe). Coronal T2-weighted image showing enlargement of interfolium spaces in the upper part of the cerebellar hemispheres. e STXBP1: P6 (4 years): axial T2-weighted and coronal fluid attenuation inversion recovery (FLAIR) sequences showing a normal cerebellum. $\mathbf{f}$ CACNA1A: P16 (4 years): midsagittal and coronal T2-weighted sequences showing enlargement of the interfolium spaces, affecting predominately the anterior lobe of the vermis. g AP4M1: P10 (18 years): midsagittal T2-weighted and coronal IR sequences showing the cerebellar vermis with an enlargement of the interfolium spaces (rostral part of the posterior lobe) and an enlarged primary fissure. The lateral part of the cerebellar hemispheres presents an enlargement of the interfolium. The hemispheric white matter volume is abnormally reduced and associated with asymmetric ventricular dilatation and thinning of the posterior part of the corpus callosum. $\mathbf{h}$ BRAT1: P9 (4 years): midsagittal T1- and coronal T2-weighted sequences showing a small vermis and diffuse enlargement of interfolium spaces of the entire cerebellum. i CACNA2D2: P12 (18 years): midsagittal T1-and coronal T2-weighted sequences showing a small vermis with enlargement of the interfolium spaces of the anterior lobe and an enlarged primary fissure. The cerebellar hemispheres are normal. j NGLY1: P10 (5 years): midsagittal T1weighted and coronal T2-weighted sequences showing slight dilatation of the left ventricle with a normal posterior fossa. k PIGS: P14 (3 years): midsagittal T1- and coronal T2-weighted sequences showing diffuse enlargement of the interfolium spaces of the entire cerebellum. I SKOR2: P15 (10 years): midsagittal T1-weighted sequences showing a small pons and a small vermis, and coronal T2-weighted sequences showing abnormal foliation and small hemispheres. Note that the entire posterior fossa seemed to be small with verticalization of the tentorium.

tremor, ataxia, and an absence of cerebellar atrophy, ${ }^{19}$ but pathogenic variants have recently been associated with the "ataxia-tremor-retardation" phenotype without noticeable epilepsy. ${ }^{18}$ Because the description of this phenotype is recent, we aimed to assess its frequency in our cohort of 180 CA patients and we identified a de novo pathogenic variant in 4 further patients. All had common features with P6, including CA, intense tremor, absent or paucisymptomatic epilepsy, and normal MRI without cerebellar atrophy (Table S4).

\section{Phenotypic expansion for four known disease genes (BRAT1, AP4M1, CACNA2D2, and NGLY1)}

Seven of the 20 patients (35\%) carried variants of four genes implicated in another neurological phenotype (Table 1, Table S3 for ACMG/AMP classification, Fig. S1). We identified the same homozygous variant of the BRAT1 (BRCA1-associated ATM activator 1) gene, resulting in an in-frame deletion of two amino acids (NM_152743.3: c.925_930del; p.Pro309_Gln310del), in three unrelated Tunisian patients (P7, P8, and P9). BRAT1 sequencing in our replication cohort detected another two unrelated patients harboring compound heterozygous variants including the c.638dupA variant, previously reported in the homozygous state in patients with rigidity and multifocal seizure syndrome, lethal neonatal (RMFSL, MIM 614498) 20. This variant was in trans with another variant, different in each patient, novel, and classified as likely pathogenic (p.Pro323Leu and p.Ala164Val). The two patients with the c.638dupA variant had intractable seizures, but none of the three patients with the homozygous in-frame deletion had epilepsy. The variant c.925_930del was absent in 200 control chromosomes from the same geographical origin. The phenotype of these three patients, harboring a hypomorphic pathogenic variant, combines CA, ID, and nonspecific cerebellar atrophy (Fig. 1). It is at the mildest end of the spectrum ranging from the severe RMFSL to CA, and including a recently described intermediate phenotype without early lethality. ${ }^{20,21}$

An AP4M1 homozygous splice variant predicted to lead to exon 14 skipping (NM_004722.3:c.1137+1G>T) was identified in patient $\mathrm{P} 11$, who did not present the congenital spastic tetraplegia associated with the same variant in a previously described consanguineous Moroccan family. ${ }^{22}$ She had only pyramidal signs in addition to CA at 16 years of age (Table 1 ). Her two siblings, presenting the same AP4M1 PV, had a classical phenotype of spastic paraplegia and ID, but without gait ataxia. MRI revealed similar supratentorial features, but cerebellar abnormalities were present in only one of the siblings (Fig. 1). Our patient is thus the first AP4M1 patient with initial cerebellar symptoms but no early spasticity to be described.

In patient $\mathrm{P} 12$, affected with $\mathrm{CA}$ without ID and with only one febrile seizure, we identified a new CACNA2D2 homozygous variant (NM_001174051.2:c.2992G>A; p.Asp998Asn) (Table 1, Fig. 1, Fig. S2). Homozygous CACNA2D2 variants have been identified in two families with EIEE, intractable epilepsy, dyskinesia, severe motor and cognitive disabilities, and cerebellar atrophy. ${ }^{23,24}$ Another homozygous missense variant of CELSR3 segregated with the disease in both families but the authors concluded that this finding was incidental, based on functional assays and animal models of CACNA2D2 PV. Our patient had no rare pathogenic variant of CELSR3. His homozygous CACNA2D2 variant, unknown in gnom $A D$, located at the last nucleotide of exon 35, was predicted to affect the splicing of exon 35 and potentially activate a cryptic donor site, resulting in the insertion of 4 intronic nucleotides in exon 35. Reverse transcription polymerase chain reaction (RT-PCR) analysis showed the presence of two types of transcripts in the patient: wild-type and transcript with retention of 4 intronic nucleotides, predicted to produce a truncated protein. So, the milder phenotype of our patient may be explained by the production of significant amounts of the wild-type transcript (Fig. S2). However, we cannot exclude 


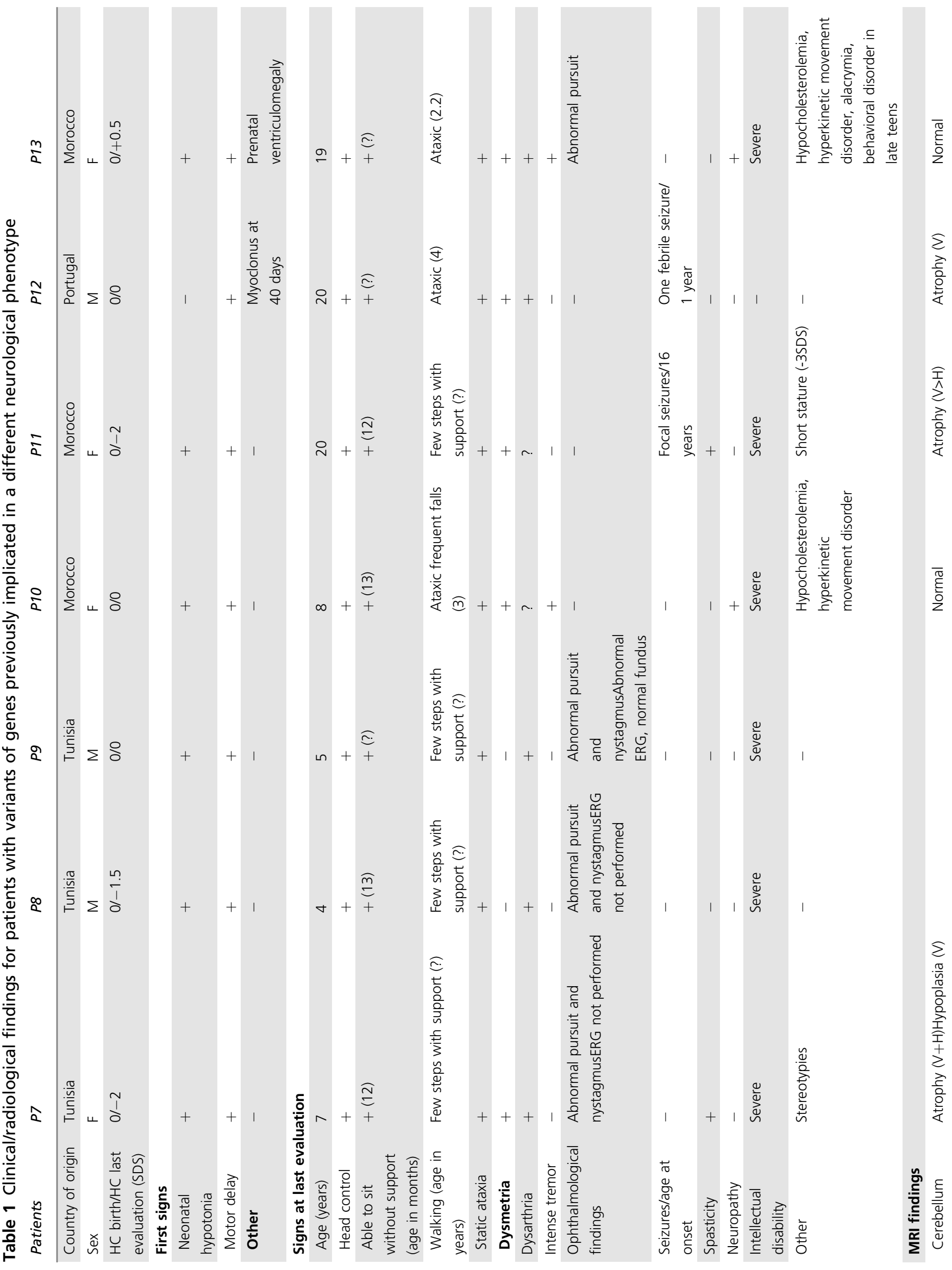




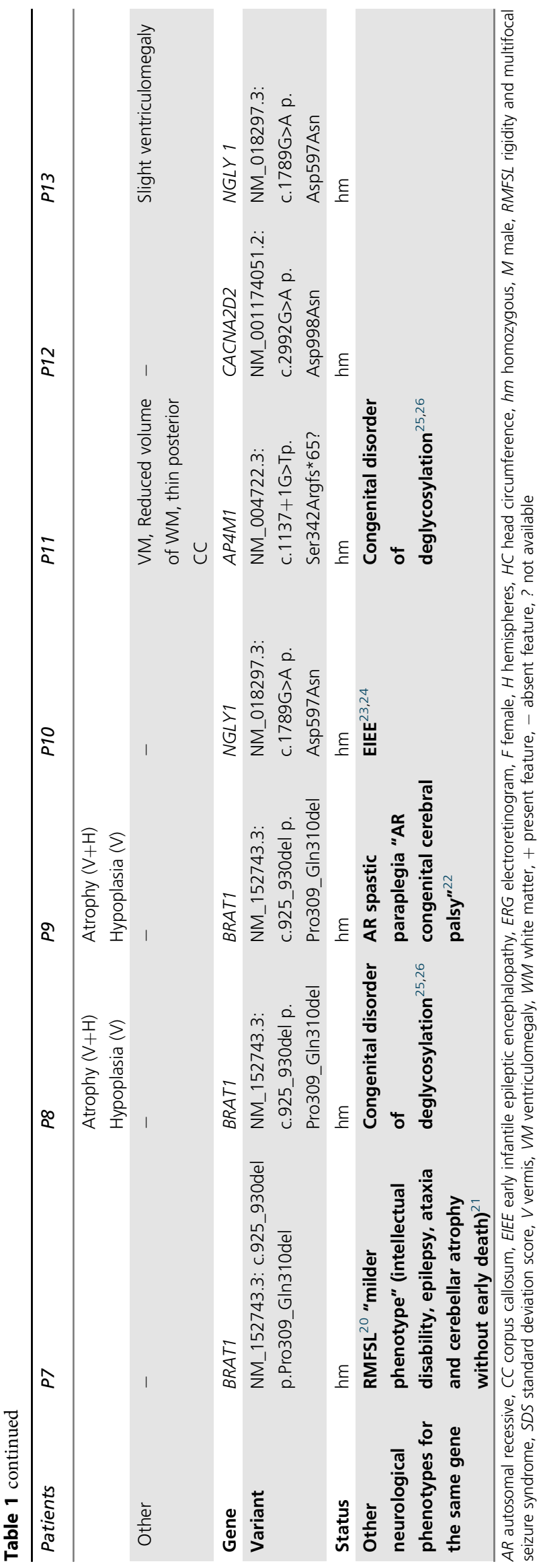

the possibility that the previously reported more severe EIEE phenotype was due to association with the CELSR3 variant, acting as a modifier gene.

A homozygous NGLY1 variant, although very rare in gnomAD (MAF 0.0002586, no homozygous), was identified in two unrelated patients (P10 and P13). This variant (NM_018297.3:c.1789G>A; p.Asp597Asn) is located at the last nucleotide of exon 13 and is predicted to alter exon splicing (MaxEntScan score $-43.7 \%$ ), and to result in a truncated protein. Recessive PV of NGLY1 gene, encoding Nglycanase, a cytoplasmic component of the endoplasmic reticulum-associated degradation pathway, cause a congenital disorder of deglycosylation (CDDG, MIM 615273) 25,26. Retrospectively, our two patients had a constellation of subtle clinical features compatible with this diagnosis: neurodevelopmental delay, hypotonia, complex hyperkinetic movement disorder, subclinical neuropathy, hypocholesterolemia, and normal MRI (Fig. 1). However, in addition to these features reported for other patients with NGLY1 amorphic PV, our patients didn't have epilepsy, abnormal liver function, ophthalmologic anomalies, or clinical regression at the time of inclusion, and they presented CA as a major symptom (Table 1).

\section{Two new CA candidate genes}

In patients P14 and P15, only one of the rare homozygous variants identified by ES in each case could convincingly account for the phenotype. Patient P14 harbored a homozygous variant of PIGS (NM_033198.3:c.91G>A; p.Gly31Arg; unknown in gnomAD; PolyPhen-2: probably damaging score 1.000; SIFT: deleterious; MutationTaster: disease-causing) (Fig. 2, Table S3). The first symptoms were psychomotor delay and several seizures at 6 months of age without recurrence. At age 10, she had a nonprogressive ataxia and dysarthria, was able to walk only with support, and had strabismus, chronic diarrhea, normal occipitofrontal circumference (OFC) and moderate intellectual deficiency. Metabolic screening and serum phosphatase alkaline were normal. The PIGS gene encodes a protein involved in glycosylphosphatidylinositol (GPI)-anchor biosynthesis. The GPIanchor is a glycolipid that serves to anchor proteins to the cell surface and is critical for many cell functions, including embryogenesis and neurogenesis. ${ }^{27,28}$ The posttranslational modification that led to the attachment of a GPI-anchor to a protein occurs in the endoplasmic reticulum and needs 10 successive stages. PIGS is one of five components of the (GPI) transamidase complex, which mediates transfer of the anchor to the protein at the end of the biosynthesis. PIGS variants have never been associated with human disorders, but 13 other genes from the same pathway cause inherited autosomal recessive GPI deficiencies (IGDs). ${ }^{27}$ IGDs are characterized by multiple organ anomalies, neuromotor impairment, ID, frequent epilepsy, and cerebellar atrophy in some cases. ${ }^{29-32}$ Patients with IGDs have low levels of various GPI-anchored cell-surface proteins (GPI-Aps), which can be diagnosed by quantification with multiparameter flow 
a

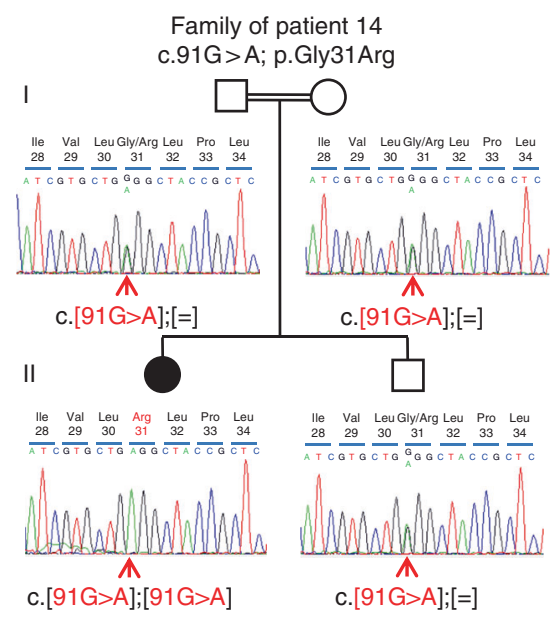

b

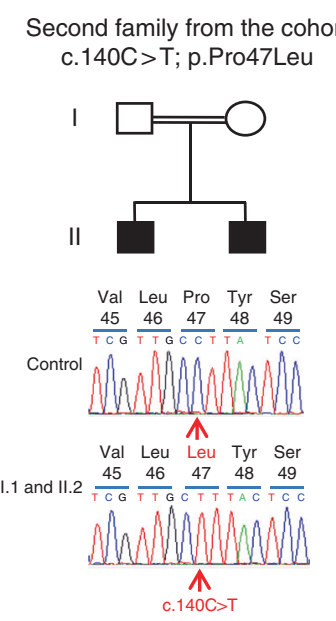

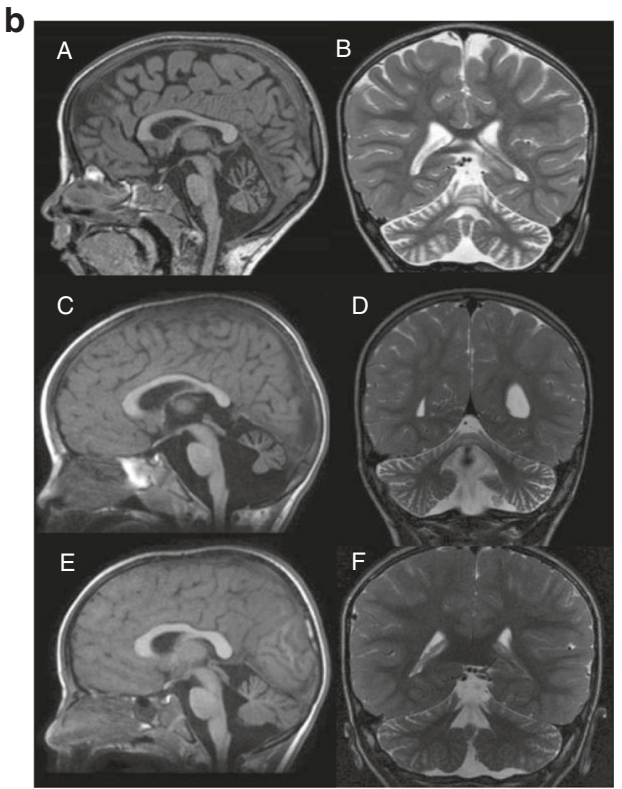

C

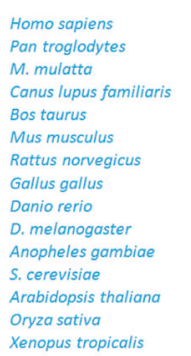

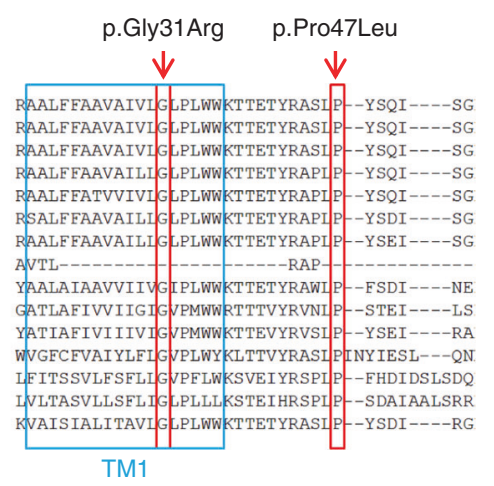

d
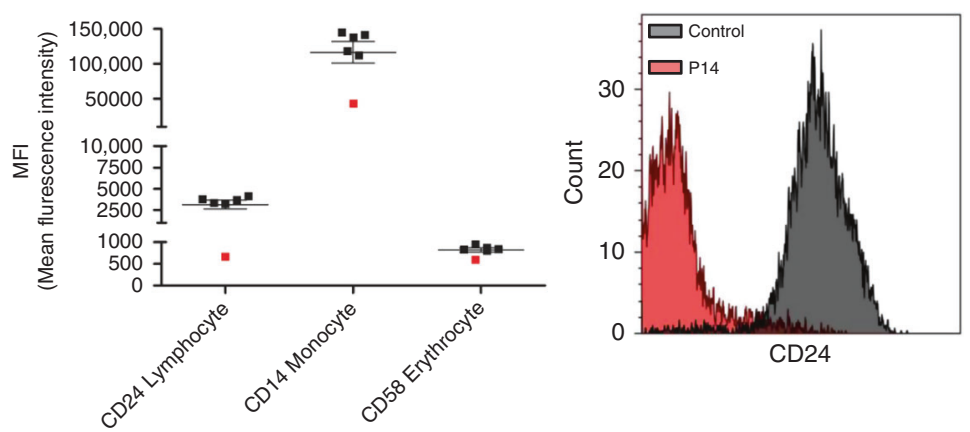

Fig. 2 Identification of likely pathogenic variants in PIGS. a Pedigrees of the 2 families and electrophoregrams of the index cases, parents, and sibs when available. b Brain magnetic resonance image (MRI) of patients with PIGS variants: A, B: P14 (3 years): midsagittal T1- and coronal T2-weighted sequences showing diffuse enlarged folium of the entire cerebellum with a slight enlargement of the fourth ventricle. C, D, E, F: MRI of two further sibs (from the replication cohort) with a PIGS likely pathogenic variant. C, D: Younger brother at 2 years: midsagittal T1- and coronal T2-weighted sequences showing diffuse enlarged folium of the entire cerebellum and marked enlargement and opening of the fourth ventricle. E, F: Older brother at 4 years: midsagittal T1- and coronal T2-weighted sequences showing slight enlarged folium of the entire cerebellum with a slight enlargement of the fourth ventricle. $\mathbf{c}$ Evolutionary conservation of the two amino acids affected by the substitution variants. The positions of the variants are indicated in red. The blue rectangle shows the position of the first transmembrane domain. $\mathbf{d}$ Flow cytometry of GPI-linked antigens (fresh blood). The left panel reports quantitative data of cell surface expression for $3 \mathrm{GPI}$-anchored proteins (CD24, CD14, and CD58). Black squares represent the mean fluorescence intensity (MFI) in the 5 healthy controls children (means \pm SD) and red squares represent the MFI in patient P14. CD24, CD14, and CD58 expression was respectively decreased in patient P14 by $5.49,2.99$, and 1.44 as compared with the mean $(n=5)$ of normal samples. The right panel illustrates overlay of CD24 expression on normal B lymphocytes (black histogram) and on P14 B lymphocytes (red histogram)

cytometry. We tested our patient and we observed lower levels of GPI-Aps expression in his lymphocytes than in the controls (Fig. 2). PIGS sequencing in the replication cohort identified a homozygous missense variant (NM_033198.3:c.140C>T; p. Pro47Leu; allelic frequency in gnomAD: $0.002165 \%$, no homozygous; PolyPhen-2: probably damaging score 1.000; SIFT: deleterious; MutationTaster: disease-causing) in 2 siblings with CA. Both had ataxia, language delay, mild ID, normal OFC, and progressive cerebellar atrophy (Fig. 2). The youngest was able to stand with support at 4 years. At age 7 , the oldest was able to walk unsteadily without support, and to read and write a few words. Previous metabolic and genetic analyses in these patients have been negative (32 ataxia genes including the genes described in this report and the genes reported in Table S1). Unfortunately, we were unable to get a new fresh blood sample from these two affected brothers to perform the study of GPI-Aps. The two missense identified in the two families are predicted to be deleterious by the three in silico tools. They are very close to each other and are located in a highly conserved region of the protein, one of them being in the first transmembrane domain of the protein. The recurrence with a concordant phenotype and an 
a
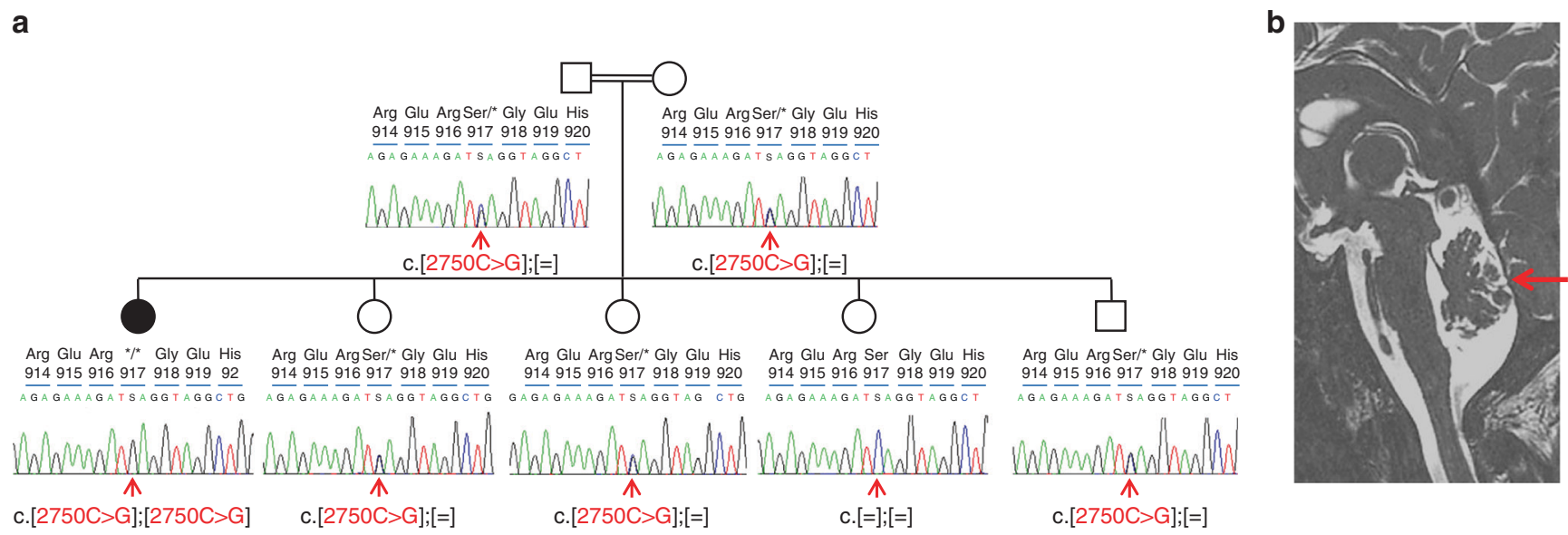

Fig. 3 SKOR2 pathogenic variant in patient 15. a Pedigree of the family of patient 15 and segregation of the pathogenic p.Ser917* variant identified in SKOR2. P15 is indicated by filled symbol. b Brain posterior fossa of P15 showing peculiar vermian dysplasia (abnormal foliation, mainly in lobules IV-VIII: red arrow) consistent with the murine model described by Wang et al. ${ }^{33}$

abnormal flow cytometry profile in the first patient are strong claims in favor of the causality of the two new PIGS variants in the phenotype.

Finally, we identified a homozygous null variant of SKOR2 in patient P15 (NM_001278063.1:c.2750C $>$ G; p.Ser917*) (Fig. 3, Table S3). The patient, a 10-year-old female, was born from consanguineous Turkish parents, and had four healthy siblings. First symptoms were neonatal hypotonia and psychomotor delay. She was able to walk independently at age 2 years, but had an ataxic gait. At age 10, she had scoliosis, nystagmus, mild ID, and a nonprogressive ataxia. MRI showed a thick corpus callosum, a small posterior fossa, a thin pons, and a unique pattern of cerebellar dysplasia, with predominance of the abnormal foliation in vermian lobules IV to VIII (Fig. 3). Segregation analysis of the variant in the family showed that both parents were heterozygous and no healthy sibling was homozygous (Fig. 3). SKOR2, encoding SKI family transcriptional corepressor 2, has never been implicated in human disorders, but is a very good candidate gene for cerebellar ataxia with cerebellar underdevelopment. Indeed, it is strongly expressed in the central nervous system, particularly in Purkinje cells and its loss of function causes ataxia in mice, leading to a small cerebellum and abnormal foliation, with a pattern very similar to that observed in patient P15, as shown by Wang et al. in Fig. 2 of their article. ${ }^{33,34}$ We didn't observe such a radiological phenotype in our cohort, suggesting that it is very rare, and actually, targeted sequencing on all exons of SKOR2 in the replication cohort found no other occurrence of SKOR2 PV.

\section{Patients without convincing causal variants}

We failed to identify any convincing variant in four patients (20\%). Clinical/radiological CA phenotypes were unremarkable in all but one of these patients, who had ptosis and bilateral oculomotor nerve palsy, suggesting a mitochondrial disease without biochemical markers (Table S5).

\section{DISCUSSION}

We report the results of ES for $20 \mathrm{CA}$ patients from independent, consanguineous families recruited at the French Reference Centre for Cerebellar Malformations and Congenital Diseases. We were able to identify causal or strong candidate variants in 16 of the 20 families (80\%) by singleton ES, including 12 identified by a filtering strategy based primarily on homozygosity mapping. Singleton ES is less expensive than trio analysis, but one of the pitfalls of this strategy is the possibility of missing de novo PV. We overcame this problem by carefully examining heterozygous variants, at least for dominant or X-linked genes associated with $\mathrm{CA}$, and we detected pathogenic de novo variants in four patients (genes ITPR1, CACNA1A, and STXBP1).

This study is unique in its focus on a reasonably homogeneous nosological entity, based on the clinical definition of CA rather than the use of cerebellar atrophy as the major inclusion criterion. Most previous reports included patients with ataxia and/or cerebellar atrophy, regardless of age at onset, and did not distinguish between clearly progressive and apparently nonprogressive ataxia. ${ }^{6,8-10,35}$ All our patients seemed to be stable at inclusion (ages 4 to 20), but we identified pathogenic variants in SACS in a 12-year-old female and in SNX14 in a 10-year-old male, highlighting the difficulty of distinguishing between CA and very early-onset slowly progressive ataxia in young children. As this point is a major concern in our daily practice, we propose to individualize this entity that we name VEOPA (very earlyonset slowly progressive ataxia). When managing patients with a CA phenotype, only the evolution of the symptoms over several years, either the identification of a specific gene, allow them to be classified among VEOPA or CA. That is why, for the genetic diagnosis in our pediatric or young adult patients, we study both entities together (CA/VEOPA).

A retrospective review of the phenotype of our patients revealed suggestive clinical/radiological features for four genes 
(SACS, NGLY1, SNX14, and AP4M1), highlighting the diagnostic importance of extraneurological findings and brain MRI data. Conversely, we also identified variants of known CA genes with no specific hallmark in our patients, such as WDR81, CACNA1A, and ITPR1.

CACNA1A gene, which encodes a calcium channel, has been shown to be responsible for a congenital type of cerebellar ataxia as well as (between others) for early infantile epileptic encephalopathy (EIEE42). Similarly, our study expands the phenotypic range of three other genes associated with EIEE (CACNA2D2, BRAT1, and STXBP1), highlighting a close pathogenic mechanism in CA and EIEE, parts of the same spectrum, at least in some cases. This link is also suggested by the presence of clinical cerebellar signs and cerebellar atrophy in patients affected with some EIEE (KCNA2 (ref. ${ }^{36}$ ), PIGA, ${ }^{30}$ etc.). Our patients harboring PV of these genes previously associated with a severe neurological phenotype had early-onset, nonprogressive ataxia, without substantial epilepsy. These phenotypic discrepancies may reflect the hypomorphic nature of the $\mathrm{PV}$, leading to a milder phenotype in our patients, as observed for the CACNA2D2 splice PV, which leads to the production of residual amounts of wild-type transcript. The genotype-phenotype correlation is more marked in patients with BRAT1 PV: homozygous null variants result in a severe RMFSL phenotype, whereas compound heterozygosity for null/hypomorphic variants is associated with a milder phenotype. We describe here patients with homozygous hypomorphic variants responsible for an even milder phenotype without epilepsy, at the extreme end of the clinical spectrum associated with BRAT1 variants.

Finally, we identified variants of two new candidate disease genes. The protein PIGS is involved in late stages of GPIanchor biosynthesis, and is, with PIGT, PIGK, PIGU, and GPAA1, one of the proteins of the transamidase complex. The clinical phenotype reported in IGDs varies, combining ID, epilepsy, coarse facial features, and multiple organ abnormalities. MRI data are scarcely reported, but cerebellar atrophy has been described in some IGD patients, including patients with PV in GPAA1 and PIGT, the two other components of the transamidase complex known in human pathologies. ${ }^{29-32}$ In addition to ID, CA with cerebellar atrophy was the only major symptom in our three patients. Because the identified variants are homozygous missense, and despite the recurrence with a concordant phenotype and GPI-Aps anomalies in the first patient, we assume that further patients are needed to formally confirm that the spectrum of IGDs expands to CA.

In one patient with an unusual dysplastic vermis, we identified a homozygous nonsense variant of SKOR2, a gene never implicated in human disorders before. Mice with a Skor2 loss of function display defective Purkinje cell development and their granule cell proliferation is highly impaired. Correct cerebellum development requires coordinated sonic hedgehog (Shh) signaling from Purkinje to granule cells. Wang et al. showed that Skor2 regulated Shh expression in Purkinje cells. ${ }^{33}$ We assume that SKOR2 is a good candidate gene for the cerebellar developmental disease of our patient, but we need to identify additional CA patients with SKOR2 pathogenic variant to draw firm conclusions about the role of this gene in the disease. Our results suggest that SKOR2 should be analyzed in CA patients, particularly those with a dysplasia predominantly affecting vermian lobules IV-VIII.

Our study illustrates the diversity of the pathophysiological pathways that may be involved in CA/VEOPA: channelopathies (CACNA2D2, CACNA1A), intracellular calcium release proteins (ITPR1), molecular chaperones (SACS), intracellular trafficking (SNX14), release of neurotransmitters from synaptic vesicles (STXBP1), sonic hedgehog pathway (SKOR2), GPI-anchor biosynthesis (PIGS), and cellular response to DNA damage (BRAT1). Although the pathological link between these pathologies is not evident at first sight, several of them are supposed to impact either development, or function/survival of Purkinje cells, the sole output neurons of the cerebellum cortex. SNX14 PV have been shown to alter Purkinje cell survival. ${ }^{14}$ It is well known that ion channels play a major role in the cerebellum and that channels anomalies are responsible for Purkinje dysfunction. ${ }^{37}$ Other anomalies of proteins involved in CA may act via channel dysfunction, like ITPR1, which mediates $\mathrm{Ca} 2$ release from the endoplasmic reticulum in various neurons, including Purkinje neurons. It is noteworthy that the $\alpha 2 \delta-2$ subunit encoded by the CACNA2D2 gene is a GPI-anchored protein, suggesting that this family of voltage-gated calcium channels may play a role in the cerebellar impairment observed in IGD patients. $^{38}$ On the other side, SKOR2 is a Purkinje cell progenitor marker, and anomalies of this gene are supposed to alter development of these cerebellar neurons. $^{39}$

ES analysis failed to identify convincing causal variants in four patients. Further analysis in these patients will probably require the genome sequencing of trios to explore deep introns or promoters, and not miss de novo variants in new or nonclassical CA-associated genes. Alternatively, in the short term, a reanalysis of our exome data may lead to identification of the causal variant in the light of new knowledge.

In conclusion, our study confirms the considerable genetic and pathophysiological heterogeneity of CA. In addition to a high rate of molecular diagnosis and identification of two new candidate genes, we highlight the lumping together of some EIEE and CA cases in the same pathophysiological pathways.

\section{ACKNOWLEDGEMENTS}

This work was supported by the Ministère de la Santé (PHRC 2009, Grant-AOM 09178), DHOS (array-CGH analysis), the association "Connaître les Syndromes Cérébelleux" (CSC), and the Fondation Maladies Rares. We thank the patients and their families for their participation in the study. We thank Anne Legall, Pascaline Berthomé, and Cindie Silva for molecular analysis. We are grateful to Johana Afenjar for her help in the drafting of the manuscript. 


\section{ELECTRONIC SUPPLEMENTARY MATERIAL}

The online version of this article (https://doi.org/10.1038/s41436018-0089-2) contains supplementary material, which is available to authorized users.

\section{REFERENCES}

1. Batten FE. Congenital cerebellar ataxia. Clin J. 1903;22:81.

2. Batten FE. Ataxia in childhood. Brain. 1905;28:484.

3. Ingram TTS. Congenital ataxic syndromes in cerebral palsy. Acta Paediatr. 1962;51:209-21.

4. Harding AE. Early onset cerebellar ataxia with retained tendon reflexes: a clinical and genetic study of a disorder distinct from Friedreich's ataxia. $J$ Neurol Neurosurg Psychiatry. 1981;44:503-8.

5. Steinlin M. Non-progressive congenital ataxia. Brain Dev. 1998;20:199-208.

6. Al-Maawali A, Blaser S, Yoon G. Diagnostic approach to childhood-onset cerebellar atrophy: a 10-year retrospective study of 300 patients. J Child Neurol. 2012;27:1121-32.

7. Poretti A, Wolf $\mathrm{NI}$, Boltshauser E. Differential diagnosis of cerebellar atrophy in childhood: an update. Neuropediatrics. 2015;46:359-70.

8. Ohba C, Osaka H, lai M, et al. Diagnostic utility of whole exome sequencing in patients showing cerebellar and/or vermis atrophy in childhood. Neurogenetics. 2013;14:225-32.

9. Fogel $\mathrm{BL}$, Lee $\mathrm{H}$, Deignan $\mathrm{JL}$, et al. Exome sequencing in the clinical diagnosis of sporadic or familial cerebellar ataxia. JAMA Neurol. 2014;71:1237-46

10. Pyle A, Smertenko T, Bargiela D, et al. Exome sequencing in undiagnosed inherited and sporadic ataxias. Brain J Neurol. 2015;138:276-83.

11. Landrum MJ, Lee JM, Benson $M$, et al. ClinVar: public archive of interpretations of clinically relevant variants. Nucleic Acids Res. 2016;44:862-8.

12. Richards $S$, Aziz N, Bale $S$, et al. Standards and guidelines for the interpretation of sequence variants: a joint consensus recommendation of the American College of Medical Genetics and Genomics and the Association for Molecular Pathology. Genet Med. 2015;17:405-24.

13. Gulsuner S, Tekinay AB, Doerschner K, et al. Homozygosity mapping and targeted genomic sequencing reveal the gene responsible for cerebellar hypoplasia and quadrupedal locomotion in a consanguineous kindred. Genome Res. 2011;21:1995-2003.

14. Akizu N, Cantagrel V, Zaki MS, et al. Biallelic mutations in SNX14 cause a syndromic form of cerebellar atrophy and lysosome-autophagosome dysfunction. Nat Genet. 2015;47:528-34.

15. Pilliod J, Moutton S, Lavie J, et al. New practical definitions for the diagnosis of autosomal recessive spastic ataxia of Charlevoix-Saguenay. Ann Neurol. 2015;78:871-86.

16. Huang L, Chardon JW, Carter MT, et al. Missense mutations in ITPR1 cause autosomal dominant congenital nonprogressive spinocerebellar ataxia. Orphanet J Rare Dis. 2012;7:67.

17. Travaglini L, Nardella M, Bellacchio $E$, et al. Missense mutations of CACNA1A are a frequent cause of autosomal dominant nonprogressive congenital ataxia. Eur J Paediatr Neurol. 2017;21:450-6.

18. Gburek-Augustat J, Beck-Woedl S, Tzschach A, et al. Epilepsy is not a mandatory feature of STXBP1-associated ataxia-tremor-retardation syndrome. Eur J Paediatr Neurol. 2016;20:661-5.

19. Di Meglio C, Lesca G, Villeneuve N, et al. Epileptic patients with de novo STXBP1 mutations: Key clinical features based on 24 cases. Epilepsia. 2015;56:1931-40.
20. Saunders CJ, Miller NA, Soden SE, et al. Rapid whole-genome sequencing for genetic disease diagnosis in neonatal intensive care units. Sci Transl Med. 2012;4:154ra135.

21. Srivastava $\mathrm{S}$, Olson HE, Cohen JS, et al. BRAT1 mutations present with a spectrum of clinical severity. Am J Med Genet A. 2016;170:2265-73.

22. Verkerk AJMH, Schot R, Dumee B, et al. Mutation in the AP4M1 gene provides a model for neuroaxonal injury in cerebral palsy. Am J Hum Genet. 2009:85:40-52.

23. Edvardson S, Oz S, Abulhijaa FA, et al. Early infantile epileptic encephalopathy associated with a high voltage gated calcium channelopathy. J Med Genet. 2013;50:118-23.

24. Pippucci $T$, Parmeggiani $A$, Palombo $F$, et al. A novel null homozygous mutation confirms CACNA2D2 as a gene mutated in epileptic encephalopathy. PLOS ONE. 2013;8:e82154

25. Enns GM, Shashi $V$, Bainbridge $M$, et al. Mutations in NGLY1 cause an inherited disorder of the endoplasmic reticulum-associated degradation pathway. Genet Med. 2014;16:751-8.

26. Lam C, Ferreira $C$, Krasnewich $D$, et al. Prospective phenotyping of NGLY1-CDDG, the first congenital disorder of deglycosylation. Genet Med. 2017;19:160-8.

27. Kinoshita T. Biosynthesis and deficiencies of glycosylphosphatidylinositol. Proc Jpn Acad Ser B Phys Biol Sci. 2014;90:130-43.

28. Um JW, Ko J. Neural Glycosylphosphatidylinositol-Anchored Proteins in Synaptic Specification. Trends Cell Biol. 2017;27:931-45.

29. Krawitz PM, Schweiger MR, Rödelsperger $C$, et al. Identity-by-descent filtering of exome sequence data identifies PIGV mutations in hyperphosphatasia mental retardation syndrome. Nat Genet. 2010;42:827-9.

30. Fauth $C$, Steindl $K$, Toutain $A$, et al. A recurrent germline mutation in the PIGA gene causes Simpson-Golabi-Behmel syndrome type 2. Am J Med Genet A. 2016;170A:392-402.

31. Kvarnung $M$, Steindl $K$, Toutain $A$, et al. A novel intellectual disability syndrome caused by GPI anchor deficiency due to homozygous mutations in PIGT. J Med Genet. 2013;50:521-8.

32. Nguyen TTM, Murakami $Y$, Sheridan $E$, et al. Mutations in GPAA1, Encoding a GPI Transamidase Complex Protein, Cause Developmental Delay, Epilepsy, Cerebellar Atrophy, and Osteopenia. Am J Hum Genet. 2017;101:856-65.

33. Wang B, Harrison W, Overbeek PA, et al. Transposon mutagenesis with coat color genotyping identifies an essential role for Skor2 in sonic hedgehog signaling and cerebellum development. Development. 2011;138:4487-97.

34. Nakatani $T$, Minaki $Y$, Kumai $M$, et al. The c-Ski family member and transcriptional regulator Corl2/Skor2 promotes early differentiation of cerebellar Purkinje cells. Dev Biol. 2014;388:68-80.

35. Sawyer SL, Schwartzentruber J, Beaulieu CL, et al. Exome sequencing as a diagnostic tool for pediatric-onset ataxia. Hum Mutat. 2014;35:45-49.

36. Masnada S, Hedrich UBS, Gardella E, et al. Clinical spectrum and genotype-phenotype associations of KCNA2-related encephalopathies. Brain. 2017;140:2337-54.

37. Bushart DD, Shakkottai VG. Ion channel dysfunction in cerebellar ataxia. Neurosci Lett 2018. https://doi.org/10.1016/j.neulet.2018.02.005.

38. Davies A, Kadurin I, Alvarez-Laviada A, et al. The alpha2delta subunits of voltage-gated calcium channels form GPI-anchored proteins, a posttranslational modification essential for function. Proc Natl Acad Sci USA. 2010;107:1654-9.

39. Minaki $Y$, Nakatani $T$, Mizuhara $E$, et al. Identification of a novel transcriptional corepressor, Corl2, as a cerebellar Purkinje cell-selective marker. Gene Expr Patterns. 2008;8:418-23. 
Stéphanie Valence, $\mathrm{MD}^{1,2,3,4}$, Emmanuelle Cochet, $\mathrm{BS}^{2,5}$, Christelle Rougeot, $\mathrm{MD}^{2,6}$, Catherine Garel, MD ${ }^{2,3,7}$, Sandra Chantot-Bastaraud, MD ${ }^{2,3,5}$, Elodie Lainey, MD, PhD ${ }^{8,9}$, Alexandra Afenjar, MD 2,3,5, Marie-Anne Barthez, MD ${ }^{10}$, Nathalie Bednarek, MD, PhD ${ }^{11}$ ', Diane Doummar, MD ${ }^{1,2,3}$, Laurence Faivre, MD, PhD ${ }^{12,13,14}$, Cyril Goizet, MD, PhD ${ }^{15,16}$, Damien Haye, MD ${ }^{2,3,5}$, Bénédicte Heron, MD ${ }^{1,2,3}$, Isabelle Kemlin, , MD ${ }^{1}$, Didier Lacombe, MD ${ }^{15,16}$, Mathieu Milh, MD, PhD ${ }^{17,18}$, Marie-Laure Moutard, MD ${ }^{1,2,3}$, Florence Riant, PharmD ${ }^{19}$, Stéphanie Robin, MD ${ }^{20}$, Agathe Roubertie, $\mathrm{MD}^{21,22}$, Pierre Sarda, MD, $\mathrm{PhD}^{23}$, Annick Toutain, $\mathrm{MD}^{24,25}$, Laurent Villard, $\mathrm{PhD}^{17,26}$, Dorothée Ville, MD ${ }^{27}$, Thierry Billette de Villemeur, MD ${ }^{1,2,3}$, Diana Rodriguez, MD, PhD $1,2,3,4$ and Lydie Burglen, MD, PhD $2,3,4,5$

${ }^{1}$ APHP, GHUEP, Hôpital Armand Trousseau, Service de Neurologie Pédiatrique, Paris, France. ${ }^{2}$ Centre de Référence Maladies Rares "Malformations et Maladies Congénitales du Cervelet", Paris-Lyon-Lille, France. ${ }^{3}$ Sorbonne Université, GRC $n^{\circ} 19$, Pathologies Congénitales du Cervelet-LeucoDystrophies, APHP, Hôpital Armand Trousseau, F-75012 Paris, France. ${ }^{4}$ INSERM U1141, Université Paris Diderot, Paris, France. ${ }^{5}$ APHP, GHUEP, Hôpital Armand Trousseau, Département de Génétique Médicale, Paris, France. ${ }^{6}$ Département de Neurologie Pédiatrique et Centre de Référence Déficiences Intellectuelles, Hôpital Femme Mère Enfant, Hospices Civils de Lyon, Centre Hospitalier Universitaire de Lyon, Lyon, France. ${ }^{7}$ APHP, GHUEP, Hôpital Armand Trousseau, Service de Radiologie Pédiatrique, Paris, France. ${ }^{8}$ APHP, Hôpital Robert Debré, Service d'Hématologie Biologique, Paris, France. ${ }^{9}$ Université Paris Diderot, UMRS_1131, Institut Universitaire d'Hématologie Paris, Paris, France. ${ }^{10}$ Service de Neuropédiatrie et Handicaps, Hôpital Gatien de Clocheville, CHU Tours, France. ${ }^{11}$ Service de Néonatologie, Institut Alix de Champagne, CHU Reims, Reims, France. ${ }^{12}$ Centre de Génétique et Centre de Référence Anomalies du Développement et Syndromes Malformatifs, Hôpital d'Enfants, Centre Hospitalier Universitaire de Dijon, Dijon, France. ${ }^{13}$ Fédération Hospitalo-Universitaire Médecine Translationnelle et Anomalies du Développement (FHU TRANSLAD), Centre Hospitalier Universitaire de Dijon, Dijon, France. ${ }^{14}$ INSERM UMR1231, Génétique des Anomalies du Développement, Université de Bourgogne, Dijon, France. ${ }^{15}$ Centre de référence Neurogénétique, Service de Génétique Médicale, Centre Hospitalier Universitaire de Bordeaux, Bordeaux, France. ${ }^{16}$ Maladies Rares, Génétique et Métabolisme (MRGM), INSERM U1211, Université de Bordeaux, Bordeaux, France. ${ }^{17}$ Aix Marseille Univ, INSERM, UMR-S 1251, MMG, Marseille, France. ${ }^{18} \mathrm{AP}-\mathrm{HM}$, Département de Neurologie Pédiatrique, Hôpital de la Timone, Marseille, France. ${ }^{19}$ AP-HP, Groupe Hospitalier Lariboisière-Fernand Widal, Laboratoire de Génétique, Paris, France. ${ }^{20}$ Service de Pédiatrie, CHU de la Réunion - Hôpital Félix Guyon, Saint-Denis, France. ${ }^{21} \mathrm{CHU}$ Gui de Chauliac, Service de Neurologie Pédiatrique, Montpellier, France. ${ }^{22}$ Institut des Neurosciences de Montpellier, INSERM U1051, Université de Montpellier, Montpellier, France. ${ }^{23}$ Service de Génétique Médicale, Hôpital Arnaud de Villeneuve, Montpellier, France. ${ }^{24}$ Centre Hospitalier Universitaire de Tours, Service de Génétique, Tours, France. ${ }^{25}$ UMR 1253, iBrain, Université de Tours, Inserm, Tours, France. ${ }^{26}$ AP-HM, Département de Génétique Médicale, Hôpital de la Timone, Marseille, France. ${ }^{27}$ Département de Neurologie Pédiatrique et Centre de Référence des Epilepsies Rares, Hôpital Femme Mère Enfant, Hospices Civils de Lyon, Centre Hospitalier Universitaire de Lyon, Lyon, France 